\title{
Optical freeform generation by laser machining and plasma- assisted polishing
}

\author{
Thomas Arnold ${ }^{1,2^{*}}$, Anne Maiwald ${ }^{2}$, Georg Böhm², Martin Erhrhard², and Klaus Zimmer ${ }^{2}$ \\ ${ }^{1}$ Institut für Fertigungstechnik, Fakultät Maschinenwesen, Technische Universität Dresden, Germany \\ ${ }^{2}$ Leibniz-Institut für Oberflächenmodifizierung e.V., Permoserstr. 15, 04318 Leipzig, Germany
}

\begin{abstract}
Tailored optical freeform lenses are required for different applications. Sub-aperture deterministic machining techniques such as plasma jet machining have shown great potential to generate freeform surfaces. However, depending on the required local slopes of the surface shape geometrical limitations occur due to the lateral tool function width. In the paper an alternative approach to fabricate freeform shapes exhibiting steep local slopes is presented. A first step involves a dwell time based fs-laser ablation process to generate the surface contour on a fused silica sample. Since the resulting roughness after laser machining lies in the range of $400 \mathrm{~nm}$ RMS which does not match optical requirements a subsequent plasma jet based polishing step is performed where micro-roughness is drastically reduced to values below $0.3 \mathrm{~nm}$ RMS.
\end{abstract}

\section{Introduction}

Today, an increasing demand for complex shaped optical elements like non-standard aspheres, acylinders, or freeform elements is observed. Recent advances in optical manufacturing technologies give more and more freedom to optical system designers to implement freeform surfaces. The use of freeform elements in optical systems provides new functionalities in illumination and imaging and yields less optical surfaces and thus more compact and lightweight system designs. In cases, where highly individualized and tailored single piece optics or small batches are required, replication techniques like moulding are hardly appropriate for production. Classical manufacturing of such optics generally involves intense sub-aperture polishing after a freeform grinding process to remove sub-surface damage (SSD). Depending on the grinding process material removal of $10-15 \mu \mathrm{m}$ is necessary to get sufficient optical surface quality in the high spatial frequency range. Furthermore, sub-aperture polishing often leads to significant shape alteration, particularly in the case of significant variations of local surface curvature, which originates from the corresponding local variation of polishing removal rates. After the polishing process, surface roughness is usually within the specified range, though an unintended shape deviation of 1-2 $\mu \mathrm{m}$ often occurs. Thus, deterministic surface error corrections are required subsequently to reach the specifications, which employ cost-intensive and time-consuming sub-aperture finishing techniques.

In the case of small sized optics exhibiting diameters of less than $10 \mathrm{~mm}$ and freeform shape departures from best fit sphere of several tens of microns, grinding and polishing techniques come to geometrical limits due to the local slopes on the surface. Hence, small sized contact- less tools are preferred that are capable to meet the requirements on surface shape generation and smoothing. In the paper an alternative approach to freeform element fabrication is presented. The process chain comprises ultra-short pulse laser machining for shape generation by ablation on fused silica windows, followed by a plasma jet polishing step. This process chain is targeting the fabrication of individual small and medium-sized optics with diameters of $1 \mathrm{~mm}-25 \mathrm{~mm}$ that exhibit steep surface slopes.

\section{Experimental}

The femtosecond laser system used for surface machining is a water-cooled CPA-2001 from CLARK-MXR. Pulses with duration of less than $150 \mathrm{fs}$ at repetition rate of $1 \mathrm{kHz}$ are emitted at a wavelength of $775 \mathrm{~nm}$. For the experiments, the mean power has been adjusted by a polarization filter to approx. $50 \mathrm{~mW}$. The laser system is equipped with fixed focussing optics. For substrate manipulation a 3-axis CNC motion system is employed. The plasma jet system utilized for polishing comprises a microwave driven plasma jet source fed by inert noble gases like argon or helium. Gas temperature in the plasma can reach up to $1500{ }^{\circ} \mathrm{C}$, which is below the softening point of fused silica. The plasma jet is brought into contact to the surface and scans over the surface. The main advantage of plasma polishing technique over mechanical-abrasive polishing is that virtually no mechanical forces are applied to the surface [1]. Hence, the surface figure remains unchanged while roughness and sub-surface damage can be significantly reduced. All experiments have been conducted on optical grade fused silica windows. Surface form and roughness measurements have been performed by tactile profiler,

Corresponding author: thomas.arnold@iom-leipzig.de 
confocal microscopy, and white light interferometer microscopy (WLI), respectively.

\section{Surface machining procedure}

Laser machined areas on fused silica samples exhibiting constant depth have been plasma polished to evaluate the smoothing capability. Figure 1 shows cross section profiles after laser machining and after plasma polishing. Roughness values of $386 \mathrm{~nm}$ RMS before and $3.8 \mathrm{~nm}$ RMS after plasma polishing, respectively, have been measured indicating a significant smoothing effect. Remaining waviness in the spatial wavelength range of approx. 100 to $200 \mu \mathrm{m}$ can be attributed in parts to laser power instabilities during the machining process. The WLI measurement shown in Figure 2 proves optical quality on the micro-roughness scale.

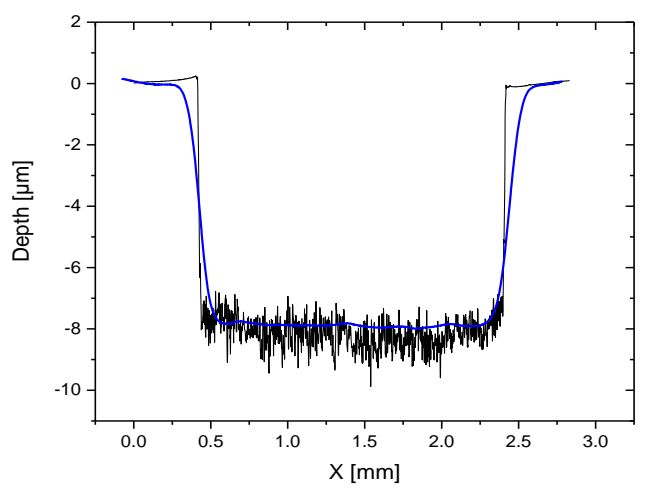

Fig. 1. Cross section profile of laser machined area before (black) and after plasma jet polishing (blue).

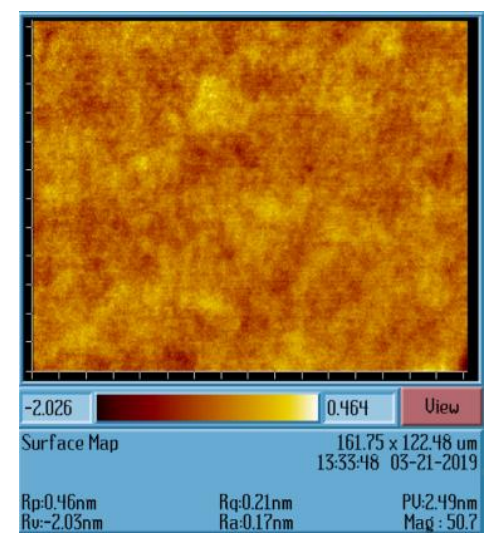

Fig. 2. Micro-roughness on plasma polished surface.

For the laser-based generation of a given surface shape, a dwell time controlled material removal is assumed. In order to determine laser machining performance with regard to ablation depth and material removal rate (MRR), a raster path motion scheme has been applied to generate area ablation of a constant depth $D$, where line feed was chosen as $d y=10 \mu \mathrm{m}$ and scan speed was varied. As can be seen from Figure 3, the removal rates calculated



Fig. 3. Material removal rates determined area ablation depending on scan velocity.

by $M R R_{\text {area }}=v \cdot D \cdot d y$ show a clear dependence on scan speed. MRR data have been fitted to an analytical expression $f(v)$. By inversion of the resulting equation a function $v(D)$ was obtained that yields local speed depending on local depth distribution $D(x, y)$. This approach is a simplified variant of the deconvolution routines normally used in dwell time procedures, e.g. in plasma jet machining [2]. Unlike methods where extensive tool functions are used, which often have a Gaussian distribution, the tool function here is considered point-like. Figure 4 shows the targeted surface profile that was to be machined in $x$ direction while the depth is constant in $y$ direction. Additionally, the obtained profile after laser machining and after plasma polishing are depicted. Since the tactile profiler only measures the upper envelope of the rough surface, the profile seems to be a bit too flat. After plasma polishing the profile is close to the target shape. The results show that a combination of laser machining and plasma polishing may be beneficial for freeform generation, especially in the case where steep local slopes are required.

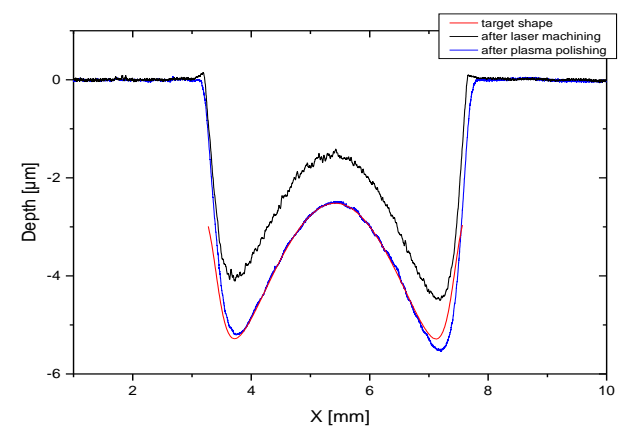

Fig. 4. Cross section profiles of targeted shape (red), after laser machining (black) and after plasma polishing (blue).

\section{References}

1. T. Arnold, G. Boehm, H. Paetzelt, J. Eur. Opt. Soc.Rapid 11, 16002 (2016)

2. T. Arnold, G. Boehm, H. Paetzelt, Proc. of SPIE Vol. 9912, 99123N-1, 2016 